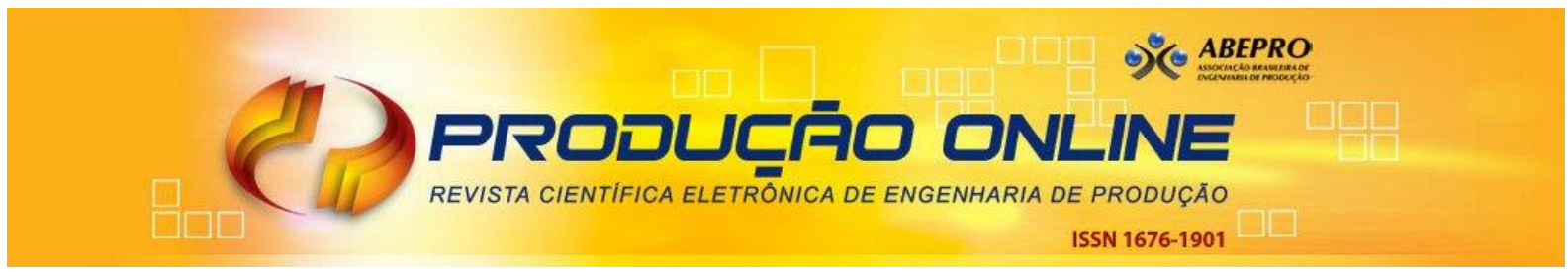

\title{
EMPREGO DE MÉTODOS DE APOIO MULTICRITÉRIO À DECISÃO PARA SELEÇÃO DE UMA ESCOLA DE IDIOMAS
}

\section{USING MULTI-CRITERIA DECISION METHODS FOR SELECTING A LANGUAGE SCHOOL}

\author{
Mileine Henriques Elias Velasco ${ }^{*}$ E-mail: mileineadm@gmail.com \\ André Luís Policani Freitas* E-mail: policani@uenf.br \\ * Universidade Estadual do Norte Fluminense Darcy Ribeiro (UENF), Campos dos Goytacazes, RJ
}

\begin{abstract}
Resumo: Atualmente, o interesse das organizações por profissionais que dominam mais de um idioma tem sido cada vez maior. Neste cenário, a escolha de uma escola de idiomas tem sido um dos problemas decisórios mais comuns, muitas vezes realizada em função de informações boca-a-boca, ações de marketing das escolas e experimentações sem critérios. Desejando contribuir para este problema, este artigo apresenta um estudo no qual foram empregados dois métodos de apoio à tomada de decisão (AHP e Média Ponderada) com o objetivo de escolher uma escola de idiomas. Para tanto, foram considerados o grau de importância de critérios relativos ao problema e o grau de satisfação de estudantes de graduação e pós-graduação em relação às escolas de idiomas que frequentavam. A escola de idiomas mais bem ordenada foi a mesma em ambos os métodos de AMD, embora algumas escolas tenham obtido posições diferentes. Foi constatado que a análise com o método AHP é mais rica e elaborada do que com a Média Ponderada. Porém, a grande quantidade de julgamentos paritários necessária ao estudo demandou atenção e significativo esforço cognitivo do decisor, além de maior tempo para realização das análises - aspectos que podem favorecer a preferência pelo uso da Média Ponderada em estudos similares.
\end{abstract}

Palavras-chave: Apoio multicritério à decisão. método de análise hierárquica. escolas de idiomas.

Abstract: Nowadays, the interest of organizations by professionals who have deep knowledge concerning more than one language has been increasing. In this scenario, the choice of a language school has been one of the most common decision problems, which has been often made on the basis of word-of-mouth, marketing activities of schools and trials without criteria. In order to contribute to this problem, this paper presents a study in which two multi-criteria decision aid methods (AHP and Weighted Average) were used to select a language school. Thus, the degree of importance of criteria relating to the problem and the degree of satisfaction of undergraduate and postgraduate students in relation to language schools they study were taken into account. The best ranked language school was the same for both MCDA methods, although some schools have obtained different positions. It was found that the analysis with AHP is richer and more elaborate than with the Weighted Average method. However, the large number of pairwise comparisons which were required to the study demanded significant attention and cognitive effort from the decision maker, and more time to perform the analysis - aspects that may contribute to the preference for the Weighted Average method in similar studies.

Keywords: Multi-criteria decision aid., Analytic Hierarchy Process. language schools. 


\section{INTRODUÇÃO}

Em um mundo globalizado as interações entre os países são cada vez maiores e, por sua vez, o interesse em pessoas que saibam lidar com essa interação também aumenta. Um dos sinais desta procura é o aumento do interesse das organizações por profissionais multilíngues, ou seja, profissionais que dominam mais de um idioma. Devido a esse fato, é percebido o aumento do interesse por escolas de idiomas.

Entretanto, atualmente existem várias escolas de idiomas (franquias ou não) presentes em todo o país, cada qual oferecendo infraestrutura, metodologias de ensino e materiais didáticos distintos, dentre outras características pertinentes ao problema. Acrescentam-se também os cursos on line (não presenciais, ministrados pela Internet) e também a maior incidência das escolas ditas "bilíngues", nas quais o ensino de idiomas é inserido na grade das disciplinas curriculares, caracterizando um ambiente altamente competitivo. Neste cenário em que múltiplas variáveis estão presentes, a discussão acerca da identificação da metodologia de ensino de idiomas mais eficiente e eficaz em termos de aprendizado não está no escopo do presente artigo. Entretanto, é muito comum que pessoas e organizações tenham dificuldade na escolha de uma escola de idiomas.

Tomar decisões é o que a maioria dos profissionais, organizações e pessoas em geral precisam fazer, em alguns casos, várias vezes ao dia. Para tanto, é necessário ter a habilidade e conhecimento para tomar decisões eficazes. A Análise Decisória Multicriterial (AMD) é uma área da Pesquisa Operacional que visa auxiliar decisores em problemas que envolvem múltiplos critérios.

Dentre os métodos de AMD mais utilizados destacam-se o método AHP (método de Análise Hierárquica) e o método da Média Ponderada. Diversas pesquisas têm utilizado o AHP como ferramenta de apoio à decisão em diversas e variadas áreas de conhecimento, como por exemplo: escolha de um Sistema integrado de Gestão (GOMES et al., 2013), mensuração do desempenho organizacional (BENTES et al., 2012), avaliação de desempenho da cadeia de suprimentos segundo critérios de competitividade empresarial (SOUZA et al., 2012), seleção de restaurante a La carte (TINOCO, 2011), e seleção de provedor de serviço logístico (LAÑEZ; CUNHA, 2006). Por outro lado, a melhoria da precisão dos modelos de previsão de desempenho de análise de sistemas com

Revista Produção Online, Florianópolis, SC, v.14, n. 4, p. 1433-1451, out./dez. 2014. 
"rajadas" de clientes simultâneos (KRISHNAMURTHY et al., 2011) e a análise do comportamento do consumidor em compras pela Internet (RATHNAM, 2005) são alguns objetivos de estudos que utilizaram a média ponderada como ferramenta de apoio à decisão.

Desejando contribuir para o problema em questão, este artigo apresenta um estudo que utilizou o método AHP e o método da média ponderada para a escolha de uma escola de idiomas. A seção 2 apresenta uma breve descrição dos dois métodos, com ênfase nas etapas do método AHP (estruturação da hierarquia, julgamentos paritários entre critérios/subcritérios, cálculo das matrizes de prioridade e verificação da consistência dos julgamentos). A seção 3 descreve o problema decisório, apresenta as etapas da aplicação dos métodos e os resultados obtidos. Finalmente, na seção 4 são apresentadas as considerações referentes ao estudo.

\section{MÉTODO DA MÉDIA PONDERADA E MÉTODO AHP}

Segundo Vinke (1989), a média ponderada é um dos métodos elementares métodos que imediatamente vêm à mente quando se confronta com um problema de agregação multicritério. O método considera o grau de importância (peso) de cada critério $j$, denotado por $p_{j}$. Dadas as avaliações de duas alternativas $a$ e $b$ à luz de $n$ critérios, denotadas por $g_{j}(a)$ e $g_{j}(b)$, o método supõe a construção de uma estrutura de preferência global representada pelo conjunto de expressões (1) de forma que:

$$
\left\{\begin{array}{l}
\text { aPb se e somente se } \sum_{j=1}^{n} p_{j} g_{j}(a)>\sum_{j=1}^{n} p_{j} g_{j}(b) \\
\text { alb se e somente se } \sum_{j=1}^{n} p_{j} g_{j}(a)=\sum_{j=1}^{n} p_{j} g_{j}(b)
\end{array}\right.
$$

O método assume que todos os critérios devem ser expressos em unidades idênticas e que as diferenças entre os valores à luz dos vários critérios podem ser compensadas. As relações de preferência, $a P b$ ( $a$ é preferível à $b$ ) e $a l b$ ( $a$ e $b$ são indiferentes) são obtidas comparando-se os valores obtidos da média ponderada de cada uma das alternativas. A alternativa que obtiver a maior média ponderada será a escolhida. 
Desenvolvido por Thomas Lorie Saaty na década de 80, o AHP (Analytic Hierarchy Process) é um método que considera elementos quantitativos e qualitativos, levando em consideração as experiências e preferências dos julgadores sobre o assunto em questão. Rafaeli e Muller (2007) definem o método AHP como um procedimento estruturado usado para a solução de problemas com diferentes variáveis que necessitam, ao mesmo tempo, serem aferidas. Quando se trata de julgamentos para se obter resultados que coincidem com a realidade, não se pode confiar somente nas pessoas, considerando que existem fatores que o homem não possui controle. Ele acaba tendendo a simplificar as coisas para tomar decisões mais rápidas. No método de análise hierárquica é importante considerar todos os fatores, mesmo que complexos (SAATY, 1980 apud LAÑEZ E CUNHA, 2006).

Para sermos realistas, nossos modelos têm de incluir e medir todos os fatores importantes, qualitativa e quantitativamente mensuráveis, sejam eles tangíveis ou intangíveis. É exatamente isto que fazemos na aplicação do método de análise hierárquica. Também consideramos as diferenças e os conflitos de opiniões como nos casos da vida real (SAATY, 1991, p.1).

O método AHP se divide em cinco etapas, sendo elas: (i) definição dos critérios, subcritérios e as alternativas; (ii) aquisição dos julgamentos comparativos, avaliação das alternativas em relação aos critérios; (iii) determinação das prioridades locais; (iv) determinação das prioridades globais das alternativas, e; (v) verificação das consistências dos julgamentos. A seguir serão explicadas essas etapas.

\subsection{Estrutura Hierárquica}

O funcionamento do método AHP está fundamentado na estrutura hierárquica dos seus dados, que é composta por vários níveis de informações, entre elas: o objetivo geral (foco principal); os critérios (também conhecidos como entidades); subcritérios (se existirem), e no alicerce da estrutura, as alternativas. Saaty (1991) define hierarquia como "um tipo de sistema particular, que é baseado no conceito de que as entidades, que tenhamos identificado, podem ser agrupadas em conjuntos distintos" e afirma que os elementos de cada nível são independentes entre si.

Além disso, é importante ressaltar que na maioria dos casos as alternativas recebem influências dos critérios, mas é possível que, em alguns desses casos, os critérios também influenciem as alternativas. Saaty (1991) denomina isso de “impacto inverso". Quando uma hierarquia é bem arquitetada ela demonstra bem a

Revista Produção Online, Florianópolis, SC, v.14, n. 4, p. 1433-1451, out./dez. 2014. 
realidade, mesmo desconsiderando os impactos inversos. A Figura 1 apresenta um exemplo de hierarquia.

Figura 1- Estrutura Hierárquica Básica

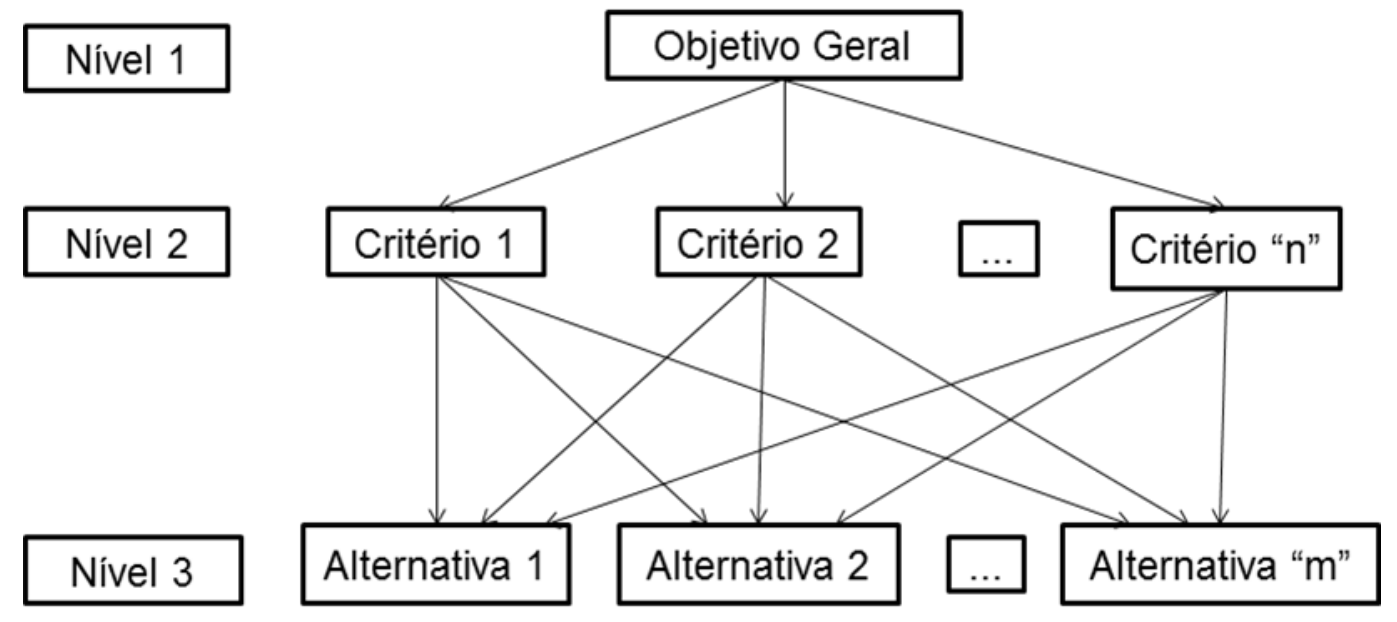

Fonte: Adaptado de Saaty (1991)

Lañez e Cunha (2006, p.398) asseguram que "esse tipo de estruturação do problema é bastante adequado, uma vez que permite uma visão bastante ampla de todo o sistema e possibilita que as influências entre os diversos elementos do problema sejam facilmente identificadas". Afirmam ainda que essa estrutura ajuda o decisor a ver toda a complexidade do problema, além de ver partes dele.

Através dessa estrutura, o decisor pode obter prioridades por meio da comparação par a par (comparação paritária) feita pelo usuário. Whyte (1969, apud Saaty 1991, p.16) confirma esta opinião, afirmando que "a abrangência da classificação hierárquica é clara. É o método mais poderoso de classificação usado pela mente humana em coordenar experiências, observações, entidades e informações". Para Saaty (1991), uma das vantagens da estrutura hierárquica é a possibilidade de encontrar o entrosamento dos níveis mais elevados da hierarquia a partir das influências mútuas entre os diversos níveis existentes na mesma hierarquia.

\subsection{Prioridade das Hierarquias}

Após a estruturação do problema, é necessário encontrar as prioridades dos elementos de um nível em relação aos de outro nível, obtidos por meio de julgamentos paritários (comparação par a par) realizado pelos julgadores, que Revista Produção Online, Florianópolis, SC, v.14, n. 4, p. 1433-1451, out./dez. 2014. 
resultará em uma matriz de decisão para cada critério ou subcritério e, dos critérios em relação ao foco principal. Para se construir uma matriz de decisão $A$, é necessária uma quantidade " $x$ " de julgamentos, que é dada pela equação (2), onde $n$ é a ordem da matriz.

$$
x=\frac{n(n-1)}{2}
$$

Nesta etapa, o julgador irá avaliar sua preferência entre os fatores, considerando os critérios no nível superior. Para tanto, ele precisará de uma escala de conversão, para atribuir valores aos seus julgamentos. A Tabela 1 apresenta a escala sugerida por Saaty (2000), conhecida na literatura como escala fundamental de Saaty.

Tabela 1 - Escala Fundamental

\begin{tabular}{|c|c|c|}
\hline $\begin{array}{c}\text { Intensidade da } \\
\text { Importância/Preferência }\end{array}$ & Definição & Interpretação \\
\hline 1 & $\begin{array}{c}\text { Igualmente } \\
\text { importante/preferível }\end{array}$ & $\begin{array}{l}\text { Os dois fatores são igualmente } \\
\text { importantes/preferíveis }\end{array}$ \\
\hline 3 & $\begin{array}{l}\text { Importância/preferência } \\
\text { moderada }\end{array}$ & $\begin{array}{l}\text { Importância/preferência moderada por } \\
\text { um dos fatores }\end{array}$ \\
\hline 5 & Importância/preferência forte & $\begin{array}{l}\text { Importância/preferência forte por um dos } \\
\text { fatores }\end{array}$ \\
\hline 7 & $\begin{array}{l}\text { Importância/preferência } \\
\text { muito forte }\end{array}$ & $\begin{array}{l}\text { Importância/preferência muito forte por } \\
\text { um dos fatores }\end{array}$ \\
\hline 9 & $\begin{array}{l}\text { Importância/preferência } \\
\text { absoluta }\end{array}$ & $\begin{array}{l}\text { Importância/preferência absolutamente } \\
\text { maior por um dos fatores }\end{array}$ \\
\hline $2,4,6$ e 8 & Valores intermediários & \\
\hline
\end{tabular}

Fonte: Adaptado de Saaty (2000)

As matrizes de julgamento devem ser normalizadas para posteriormente serem obtidas as prioridades médias locais e as prioridades globais. Saaty (1991) relaciona vários métodos para a normalização da matriz. Em um desses métodos, somam-se os valores das colunas da matriz, depois se divide cada elemento da matriz pela soma dos valores da sua própria coluna, o que resultará no quadro normalizado.

Revista Produção Online, Florianópolis, SC, v.14, n. 4, p. 1433-1451, out./dez. 2014. 
A normalização de uma matriz de julgamentos resultará em outra matriz, e para se encontrar as prioridades médias locais (PMLs) é necessário somar as linhas da nova matriz e dividir pelo número de colunas. Para se encontrar as prioridades globais (PGs) multiplicam-se as PMLs dos subcritérios à luz das alternativas pelas PMLs dos critérios em relação ao foco principal. Este cálculo é repetido com todos os critérios e alternativas da mesma linha (TREVIZANO; FREITAS, 2005).

\subsection{Consistência dos julgamentos}

É difícil garantir a consistência de medidas. Mesmo aquelas que usam instrumentos para sua medição podem ser inconsistentes, visto que pode haver erros em sua medição ou no instrumento utilizado, o que levará a conclusões erradas, principalmente se estas medidas forem muito próximas (SAATY, 1991). Em casos de julgamentos, eles também devem ser consistentes, pois é necessária a consistência para que estes sejam considerados reais. Mas quando se fala em julgamentos incluímos fatores como percepções, experiências entre outros sentimentos que levam o julgamento para a subjetividade. Nesses casos, é preciso aumentar a objetividade e diminuir a subjetividade. Saaty $(1991$, p. 11) assume que:

As comparações paritárias são obtidas por questionamento direto às pessoas (um único indivíduo, se o problema é do seu interesse apenas) que podem ou não ser especialistas, mas que estejam familiarizadas com o problema. Um ponto central em nossas abordagens é que as pessoas muitas vezes são inconsistentes, mas as prioridades têm de ser definidas, e as coisas têm de ser feitas a despeito da inconsistência.

Segundo Lañez e Cunha (2006), o conceito de consistência é oferecido através de uma quantidade de dados que são apresentados no início do processo e a partir desse processo é possível, por meio da lógica, deduzir todos os outros dados. Para eles a falta de consistência nos julgamentos não afirma que o método é falho, ela é "normal e útil", desde que controlada.

\section{A ABORDAGEM METODOLÓGICA}

Para a seleção de uma escola de idiomas foi considerado o estudo realizado por Freitas et al. (2012), que buscou mensurar a qualidade dos serviços em escolas de idiomas segundo a percepção de 133 estudantes de graduação e pós-graduação 
de uma universidade pública. No referido estudo foram consideradas dezoito escolas de idiomas atuantes em um município do Estado do Rio de Janeiro. Todas as escolas foram avaliadas à luz de quatro critérios, sendo estes subdivididos em quinze subcritérios, conforme Quadro 1.

Quadro 1 - Critérios e subcritérios considerados no estudo

\begin{tabular}{|l|l|}
\hline Critérios & \multicolumn{1}{|c|}{ Subcritérios } \\
\hline Ensino (ED) & $\begin{array}{l}\text { Didática do professor (DP), conhecimento do professor (CP), } \\
\text { evitar o uso da língua portuguesa (ELP), pontualidade (P), } \\
\text { método de ensino (ME), e qualidade do material (QM); }\end{array}$ \\
\hline Relacionamento interpessoal (RI) & $\begin{array}{l}\text { Atendimento na secretaria (AS), educação do professor (EP) e } \\
\text { convivência agradável (CA); }\end{array}$ \\
\hline Estrutura física (EF) & $\begin{array}{l}\text { Laboratório (LB), cantina (C) e sala de aula (SA), este último } \\
\text { subdividido em tamanho da sala (TM) conforto térmico (CT) e } \\
\text { conforto das cadeiras (CC). }\end{array}$ \\
\hline Valor do serviço (VS). & \\
\hline
\end{tabular}

No presente artigo foram consideradas as sete escolas de idiomas com maior número de respondentes que participaram do estudo realizado por Freitas et al. (2012), sendo estas denotadas por Escola $1\left(a_{1}\right)$, Escola $2\left(a_{2}\right), \ldots$, Escola $7\left(a_{7}\right)$. Juntas, as sete escolas correspondem a $74,4 \%$ dos respondentes.

Cada estudante estabeleceu o grau de importância de cada critério (GI) e o grau de satisfação (GS) com a sua escola de idiomas à luz de cada critério. Para tanto, foram consideradas duas escalas de avaliação não comparativas contínuas, cujos extremos eram respectivamente 0 (Nada Importante) e 100 (Muito Importante), para mensuração do grau de importância dos critérios, e 0 (Muito Insatisfeito) e 100 (Muito Satisfeito), para mensuração do grau de satisfação.

A partir dos julgamentos foi possível calcular o Grau de Importância Médio dos critérios $\overline{(G I)}$ e o Grau de Satisfação Médio dos estudantes $\overline{(G S)}$ com a escola de idiomas à luz de cada critério. Estes valores estão dispostos na Tabela 2 e foram utilizados como dados para o emprego dos dois métodos de apoio à decisão. 
Tabela 2 - Graus de Importância Médios e Graus de Satisfação Médios

\begin{tabular}{|c|c|c|c|c|c|c|c|c|c|c|c|c|c|c|c|c|}
\hline \multicolumn{2}{|c|}{ Critérios } & \multicolumn{6}{|c|}{ ED } & \multicolumn{3}{|c|}{$\mathbf{R I}$} & \multicolumn{5}{|c|}{ EF } & \multirow{2}{*}{$\begin{array}{c}\text { VS } \\
-\end{array}$} \\
\hline \multirow{2}{*}{\multicolumn{2}{|c|}{ Subcritérios }} & DP & $\mathrm{CP}$ & ELP & $\mathbf{P}$ & ME & QM & AS & EP & CA & LB & & SA & & C & \\
\hline & & & & & & & & & & & & TM & CT & CC & & \\
\hline & $\overline{G S}$ & 8,81 & 9,10 & 9,21 & 7,94 & 8,54 & 9,09 & 8,34 & 9,20 & 7,73 & 9,16 & 9,13 & 6,54 & 8,11 & 4,42 & 6,95 \\
\hline \multirow[t]{3}{*}{ Escola 1} & $\overline{G l}$ & 9,54 & 9,70 & 8,03 & 7,80 & 9,08 & 9,16 & 7,82 & 9,21 & 8,01 & 5,06 & 7,38 & 7,73 & 7,14 & 4,28 & 8,68 \\
\hline & $\overline{\overline{G I}}$ & & & & & & & & 7,91 & & & & & & & \\
\hline & $\overline{G S}$ & 8,96 & 8,44 & 9,12 & 8,84 & 8,05 & 8,48 & 7,49 & 9,10 & 7,74 & 8,89 & 8,49 & 6,34 & 7,68 & 1,97 & 6,91 \\
\hline \multirow[t]{3}{*}{ Escola 2} & $\overline{G I}$ & 9,71 & 9,31 & 8,81 & 8,35 & 9,21 & 9,26 & 8,05 & 9,20 & 7,90 & 6,23 & 7,41 & 7,65 & 7,26 & 3,38 & 7,71 \\
\hline & $\overline{\overline{G I}}$ & & & & & & & & 7,96 & & & & & & & \\
\hline & $\overline{G S}$ & 8,56 & 8,46 & 9,13 & 7,00 & 7,28 & 7,14 & 5,91 & 8,77 & 6,85 & 6,43 & 7,58 & 4,14 & 8,42 & 4,64 & 8,76 \\
\hline \multirow[t]{3}{*}{ Escola 3} & $\overline{G l}$ & 9,62 & 10,58 & 8,05 & 7,82 & 9,24 & 9,25 & 7,75 & 9,62 & 7,78 & 5,19 & 7,52 & 7,50 & 7,17 & 4,58 & 8,77 \\
\hline & $\overline{\overline{G I}}$ & & & & & & & & 8,03 & & & & & & & \\
\hline & $\overline{G S}$ & 8,77 & 8,32 & 8,33 & 7,09 & 8,40 & 7,85 & 9,36 & 9,24 & 6,12 & 8,25 & 7,85 & 4,82 & 7,31 & 5,73 & 8,09 \\
\hline \multirow[t]{3}{*}{ Escola 4} & $\overline{G l}$ & 8,52 & 10,66 & 8,10 & 7,83 & 9,30 & 9,26 & 8,26 & 8,28 & 7,54 & 4,93 & 7,55 & 7,64 & 7,14 & 4,19 & 9,07 \\
\hline & $\overline{\overline{G I}}$ & & & & & & & & 7,90 & & & & & & & \\
\hline & $\overline{G S}$ & 9,17 & 9,00 & 9,52 & 9,11 & 8,90 & 9,10 & 8,67 & 9,22 & 8,76 & 9,50 & 8,59 & 8,00 & 9,00 & 7,80 & 8,10 \\
\hline \multirow[t]{3}{*}{ Escola 5} & $\overline{G I}$ & 8,67 & 10,48 & 8,00 & 7,84 & 9,25 & 9,29 & 8,30 & 8,73 & 7,84 & 5,36 & 7,60 & 7,63 & 7,25 & 4,51 & 8,84 \\
\hline & $\overline{\overline{G I}}$ & & & & & & & & 7,97 & & & & & & & \\
\hline & $\overline{G S}$ & 7,38 & 8,01 & 8,13 & 7,30 & 7,20 & 6,80 & 7,63 & 8,60 & 7,55 & 8,75 & 7,00 & 5,91 & 8,33 & 6,19 & 7, \\
\hline \multirow[t]{3}{*}{ Escola 6} & $\overline{G l}$ & 8,28 & 10,60 & 7,90 & 7,79 & 9,24 & 9,24 & 8,31 & 8,49 & 7,66 & 5,07 & 7,50 & 7,68 & 7,22 & 4,69 & 9,13 \\
\hline & $\overline{\overline{G I}}$ & & & & & & & & 7,92 & & & & & & & \\
\hline & $\overline{G S}$ & 8,89 & 9,57 & 9,74 & 9,56 & 9,33 & 9,86 & 8,50 & 9,39 & 8,13 & 9,67 & 9,40 & 1,33 & 8,78 & 1,75 & 6,17 \\
\hline \multirow[t]{3}{*}{ Escola 7} & $\overline{G l}$ & 8,67 & 10,38 & 8,11 & 7,85 & 9,26 & 9,28 & 8,27 & 8,67 & 7,92 & 5,56 & 7,73 & 7,71 & 7,39 & 4,46 & 8,85 \\
\hline & $\overline{\overline{G I}}$ & & & & & & & & 8,01 & & & & & & & \\
\hline & $\overline{G S}$ & 8,70 & 8,99 & 7,89 & 8,23 & 8,46 & 8,46 & 7,74 & 9,01 & 8,68 & 5,60 & 8,28 & 8,61 & 7,55 & 4,83 & 7,57 \\
\hline \multirow{2}{*}{$\begin{array}{l}\text { Todos os } \\
\text { alunos }\end{array}$} & $\overline{G l}$ & 9,67 & 9,65 & 8,31 & 8,01 & 9,20 & 9,14 & 7,55 & 9,23 & 7,93 & 5,84 & 7,72 & 7,70 & 7,37 & 4,50 & 8,48 \\
\hline & $\overline{\overline{G I}}$ & & & & & & & & & & & & & & & \\
\hline
\end{tabular}

\subsection{Emprego do método AHP}

A Figura 2 ilustra a estrutura hierárquica do problema decisório em questão. É importante notar como a representação gráfica da estrutura hierárquica evidencia o relacionamento entre os elementos envolvidos (foco principal, alternativas, critérios e subcritérios), contribuindo para facilitar a compreensão do problema decisório e a sua modelagem por meio do método AHP. Entretanto, acredita-se que a representação hierárquica também seja capaz de contribuir para a modelagem de 
problemas decisórios que sejam analisados por outros métodos de apoio à decisão com múltiplos critérios.

Figura 2 - Estrutura Hierárquica do experimento

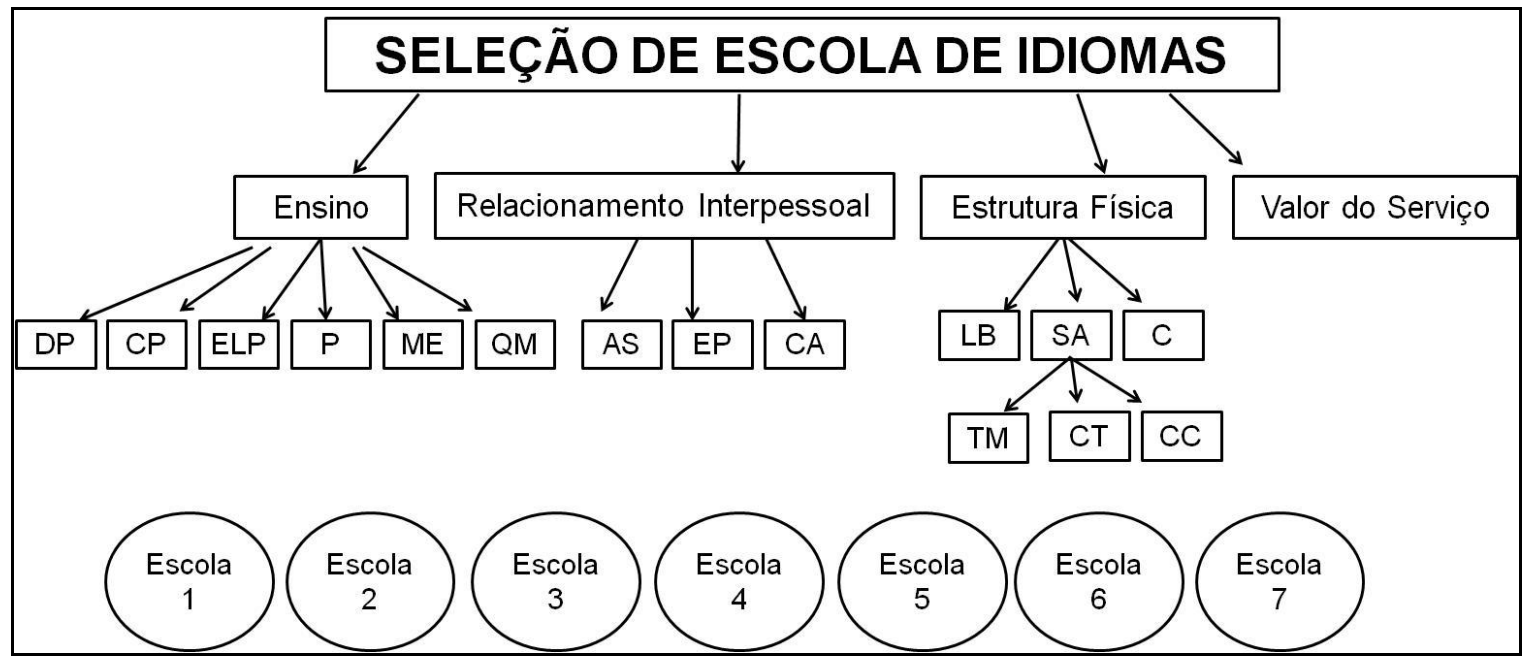

\section{- Julgamentos paritários e cálculo das Prioridades Médias Locais (PMLs)}

Os valores anteriormente apresentados na Tabela 2 foram utilizados como referência para os julgamentos paritários entre as escolas de idiomas (alternativas). Para os julgamentos paritários entre subcritérios em relação aos critérios e, entre os critérios em relação ao objetivo principal, foram utilizados os valores médios da importância segundo a percepção dos estudantes de todas as sete escolas.

$\mathrm{Na}$ Tabela 3 estão os julgamentos paritários em relação ao foco principal e as prioridades médias locais (PMLs), obtidas por meio do emprego do software IPÊ (COSTA, 2004), que implementa a lógica do método AHP. É possível observar que o ensino e estrutura física são, respectivamente, os critérios mais e menos importantes segundo a percepção dos estudantes.

Tabela 3 - Julgamentos paritários dos critérios à luz do Foco Principal

\begin{tabular}{cccccc}
\hline Foco Principal & Ensino & Relac. Interpessoal & Estrutura Física & Valor do Serviço & PMLs \\
\hline Ensino & 1 & 2 & 5 & 2 & 0,438 \\
Relac. Interpessoal & $1 / 2$ & 1 & 4 & 1 & 0,246 \\
Estrutura Física & $1 / 5$ & $1 / 4$ & 1 & $1 / 4$ & 0,070 \\
Valor do Serviço & $1 / 2$ & 1 & 4 & 1 & 0,246 \\
\hline
\end{tabular}

Revista Produção Online, Florianópolis, SC, v.14, n. 4, p. 1433-1451, out./dez. 2014. 
O mesmo procedimento foi realizado com as alternativas (escolas) em relação a cada critério/subcritério considerado, conforme mostra a Tabela 4. Por meio desta tabela, é possível constatar qual escola mais se destaca à luz de cada critério (escola que apresenta maior PML).

Tabela 4 - Prioridades Médias Locais à luz das alternativas

(Continua)

Didática do Professor $\quad$ Escola 1 Escola 2 Escola 3 Escola 4 Escola 5 Escola 6 Escola 7 PMLs

\begin{tabular}{|c|c|c|c|c|c|c|c|c|}
\hline Escola1 & 1 & 1 & 1 & 1 & 1 & 3 & 1 & 0,155 \\
\hline Escola2 & 1 & 1 & 1 & 1 & 1 & 4 & 1 & 0,161 \\
\hline Escola3 & 1 & 1 & 1 & 1 & $1 / 2$ & 3 & 1 & 0,142 \\
\hline Escola4 & 1 & 1 & 1 & 1 & 1 & 3 & 1 & 0,155 \\
\hline Escola5 & 1 & 1 & 2 & 1 & 1 & 4 & 1 & 0,181 \\
\hline Escola6 & $1 / 3$ & $1 / 4$ & $1 / 3$ & $1 / 3$ & $1 / 4$ & 1 & $1 / 4$ & 0,046 \\
\hline Escola7 & 1 & 1 & 1 & 1 & 1 & 4 & 1 & 0,161 \\
\hline Conhecimento do Professor & Escola 1 & Escola 2 & Escola 3 & Escola 4 & Escola 5 & Escola 6 & Escola 7 & PML's \\
\hline Escola1 & 1 & 2 & 2 & 2 & 1 & 3 & 1 & 0,202 \\
\hline Escola2 & $1 / 2$ & 1 & 1 & 1 & $1 / 2$ & 1 & $1 / 3$ & 0,089 \\
\hline Escola3 & $1 / 2$ & 1 & 1 & 1 & $1 / 2$ & 1 & $1 / 3$ & 0,089 \\
\hline Escola4 & $1 / 2$ & 1 & 1 & 1 & $1 / 2$ & 1 & $1 / 3$ & 0,089 \\
\hline Escola5 & 1 & 2 & 2 & 2 & 1 & 2 & $1 / 2$ & 0,172 \\
\hline Escola6 & $1 / 3$ & 1 & 1 & 1 & $1 / 2$ & 1 & $1 / 4$ & 0,081 \\
\hline Escola7 & 1 & 3 & 3 & 3 & 2 & 4 & 1 & 0,276 \\
\hline Evitar Língua Portuguesa & Escola 1 & Escola 2 & Escola 3 & Escola 4 & Escola 5 & Escola 6 & Escola 7 & PML's \\
\hline Escola1 & 1 & 1 & 1 & 2 & 1 & 3 & $1 / 2$ & 0,150 \\
\hline Escola2 & 1 & 1 & 1 & 2 & 1 & 3 & $1 / 2$ & 0,150 \\
\hline Escola3 & 1 & 1 & 1 & 2 & 1 & 3 & $1 / 2$ & 0,150 \\
\hline Escola4 & $1 / 2$ & $1 / 2$ & $1 / 2$ & 1 & $1 / 3$ & 1 & $1 / 3$ & 0,070 \\
\hline Escola5 & 1 & 1 & 1 & 3 & 1 & 3 & 1 & 0,177 \\
\hline Escola6 & $1 / 3$ & $1 / 3$ & $1 / 3$ & 1 & $1 / 3$ & 1 & $1 / 4$ & 0,056 \\
\hline Escola7 & 2 & 2 & 2 & 3 & 1 & 4 & 1 & 0,248 \\
\hline Pontualidade & Escola 1 & Escola 2 & Escola 3 & Escola 4 & Escola 5 & Escola 6 & Escola 7 & PML's \\
\hline Escola1 & 1 & $1 / 2$ & 2 & 2 & $1 / 3$ & 2 & $1 / 4$ & 0,093 \\
\hline Escola2 & 2 & 1 & 4 & 4 & 1 & 4 & $1 / 2$ & 0,196 \\
\hline Escola3 & $1 / 2$ & $1 / 4$ & 1 & 1 & $1 / 5$ & 1 & $1 / 6$ & 0,050 \\
\hline Escola4 & $1 / 2$ & $1 / 4$ & 1 & 1 & $1 / 5$ & 1 & $1 / 5$ & 0,051 \\
\hline Escola5 & 3 & 1 & 5 & 5 & 1 & 4 & $1 / 2$ & 0,224 \\
\hline Escola6 & $1 / 2$ & $1 / 4$ & 1 & 1 & $1 / 4$ & 1 & $1 / 5$ & 0,053 \\
\hline Escola7 & 4 & 2 & 6 & 5 & 2 & 5 & 1 & 0,332 \\
\hline Método de Ensino & Escola 1 & Escola 2 & Escola 3 & Escola 4 & Escola 5 & Escola 6 & Escola 7 & PML's \\
\hline Escola1 & 1 & 1 & 3 & 1 & 1 & 3 & $1 / 2$ & 0,151 \\
\hline Escola2 & 1 & 1 & 2 & 1 & $1 / 2$ & 3 & $1 / 3$ & 0,123 \\
\hline Escola3 & $1 / 3$ & $1 / 2$ & 1 & $1 / 3$ & $1 / 4$ & 1 & $1 / 5$ & 0,052 \\
\hline Escola4 & 1 & 1 & 3 & 1 & 1 & 3 & $1 / 2$ & 0,151 \\
\hline Escola5 & 1 & 2 & 4 & 1 & 1 & 4 & 1 & 0,201 \\
\hline Escola6 & $1 / 3$ & $1 / 3$ & 1 & $1 / 3$ & $1 / 4$ & 1 & $1 / 5$ & 0,049 \\
\hline Escola7 & 2 & 3 & 5 & 2 & 1 & 5 & 1 & 0,274 \\
\hline Qualidade do Material & Escola 1 & Escola 2 & Escola 3 & Escola 4 & Escola 5 & Escola 6 & Escola 7 & PML's \\
\hline Escola1 & 1 & 2 & 4 & 3 & 1 & 5 & $1 / 2$ & 0,198 \\
\hline Escola2 & $1 / 2$ & 1 & 3 & 2 & $1 / 2$ & 4 & $1 / 3$ & 0,124 \\
\hline Escola3 & $1 / 4$ & $1 / 3$ & 1 & $1 / 2$ & $1 / 4$ & 1 & $1 / 6$ & 0,045 \\
\hline Escola4 & $1 / 3$ & $1 / 2$ & 2 & 1 & $1 / 3$ & 2 & $1 / 4$ & 0,074 \\
\hline Escola5 & 1 & 2 & 4 & 3 & 1 & 5 & $1 / 2$ & 0,198 \\
\hline Escola6 & $1 / 5$ & $1 / 4$ & 1 & $1 / 2$ & $1 / 5$ & 1 & $1 / 6$ & 0,041 \\
\hline Escola7 & 2 & 3 & 6 & 4 & 2 & 6 & 1 & 0,322 \\
\hline
\end{tabular}

Revista Produção Online, Florianópolis, SC, v.14, n. 4, p. 1433-1451, out./dez. 2014. 
Tabela 4 - Prioridades Médias Locais à luz das alternativas

(Continuação)

Atendimento na Secretaria Escola 1 Escola 2 Escola 3 Escola 4 Escola 5 Escola 6 Escola 7 PML's

$\begin{array}{lcccccccc}\text { Escola1 } & 1 & 2 & 5 & 1 / 3 & 1 & 2 & 1 & 0,147 \\ \text { Escola2 } & 1 / 2 & 1 & 4 & 1 / 4 & 1 / 3 & 1 & 1 / 3 & 0,076 \\ \text { Escola3 } & 1 / 5 & 1 / 4 & 1 & 1 / 7 & 1 / 6 & 1 / 4 & 1 / 6 & 0,028 \\ \text { Escola4 } & 3 & 4 & 7 & 1 & 2 & 4 & 2 & 0,318 \\ \text { Escola5 } & 1 & 3 & 6 & 1 / 2 & 1 & 3 & 1 & 0,181 \\ \text { Escola6 } & 1 / 2 & 1 & 4 & 1 / 4 & 1 / 3 & 1 & 1 / 2 & 0,080 \\ \text { Escola7 } & 1 & 3 & 6 & 1 / 2 & 1 & 2 & 1 & 0,170\end{array}$

Educação do Professor $\quad$ Escola 1 Escola 2 Escola 3 Escola 4 Escola 5 Escola 6 Escola 7 PML's

$\begin{array}{lllllllll}\text { Escola1 } & 1 & 1 & 1 & 1 & 1 & 2 & 1 & 0,153\end{array}$

Escola2

Escola3

Escola4

Escola5

Escola6

Escola7

$\begin{array}{llll}1 & 1 & 1 & 1 \\ 1 & 1 & 1 & 1 \\ 1 & 1 & 1 & 1\end{array}$

$\begin{array}{lll}1 & 1 & 2\end{array}$

$1 \quad 0,153$

Convivência Agradável $\quad$ Escola 1 Escola 2 Escola 3 Escola 4 Escola 5 Escola 6 Escola 7 PML's

$\begin{array}{lll}1 & 1 & 1 \\ 1 & 1 & 1\end{array}$

1

$1-1$

$1 \quad 1$

2

$1 \quad 1 \quad 2$

2

1

0,153

11

$1 / 2 \quad 1 / 2$

1

$1 / 2$

$1 / 2$

2

1

0,153

$\begin{array}{lll}1 & 1 & 2\end{array}$

$\begin{array}{lll}1 & 1 & 2\end{array}$

$1 / 2$

0,086 Escola1

Escola2

$1 \quad 1$

Escola3

Escola4

Escola5

Escola6

Escola7

2

4

$1 / 3$

$1 / 3$

$1 / 4 \quad 1 / 2$

$1 / 6 \quad 1 / 3$

$1 \quad 0,136$

$\begin{array}{ll}1 / 2 & 1 / 2 \\ 1 / 4 & 1 / 4\end{array}$

Laboratório

$\begin{array}{cccc}1 / 4 & 1 / 4 & 1 / 2 & 1 \\ 3 & 3 & 4 & 6\end{array}$

$1 / 6$

1

$1 / 3 \quad 1$

$3 \quad 1 / 2$

0,136

$1 / 2 \quad 0,072$

$\begin{array}{lll}1 & 1 & 2 \\ 1 & 1 & 2\end{array}$

$\begin{array}{ll}2 & 3 \\ 2 & 5\end{array}$

$1 / 2 \quad 2$

$1 / 5$

0,038

\begin{tabular}{cc}
\hline Laboratório & Escol \\
\hline Escola1 & \\
Escola2 & \\
Escola3 & \\
Escola4 5 \\
Escola6 \\
Escola7
\end{tabular}

scola 1 Escola 2 Esco

$5 \quad 1 / 2$

$\begin{array}{ccc}1 & 1 & 6 \\ 1 & 1 & 5 \\ 1 / 6 & 1 / 5 & \\ 1 / 2 & 1 / 2 & 4 \\ 1 & 2 & 7 \\ 1 & 1 & 5 \\ 2 & 2 & 7\end{array}$

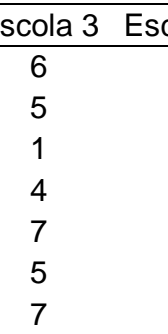

Escola 4 Esco

Escola 5 Escola

$1 / 2$

0,331

$1 / 2 \quad 0,119$

nanho da Sala

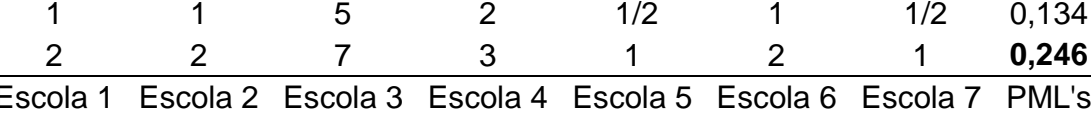

$\begin{array}{lcccccccc}\text { Escola1 } & 1 & 2 & 3 & 3 & 1 & 5 & 1 & 0,232 \\ \text { Escola2 } & 1 / 2 & 1 & 2 & 2 & 1 & 3 & 1 / 2 & 0,142 \\ \text { Escola3 } & 1 / 3 & 1 / 2 & 1 & 1 & 1 / 2 & 2 & 1 / 4 & 0,077 \\ \text { Escola4 } & 1 / 3 & 1 / 2 & 1 & 1 & 1 / 2 & 2 & 1 / 3 & 0,080 \\ \text { Escola5 } & 1 & 1 & 2 & 2 & 1 & 3 & 1 / 2 & 0,158 \\ \text { Escola6 } & 1 / 5 & 1 / 3 & 1 / 2 & 1 / 2 & 1 / 3 & 1 & 1 / 5 & 0,046 \\ \text { Escola7 } & 1 & 2 & 4 & 3 & 2 & 5 & 1 & \mathbf{0 , 2 6 5}\end{array}$

Conforto Térmico

Escola 1 Escola 2 Escola 3 Escola 4 Escola 5 Escola 6 Escola 7 PML's

Escola1

Escola2

Escola3

Escola4

Escola5

$\begin{array}{ll}1 & 1 \\ 1 & 1\end{array}$

5

$41 / 3$

$\begin{array}{ll}4 & 1 / 3 \\ 4 & 1 / 4\end{array}$

$1 / 4$

$\begin{array}{ccc}1 / 3 & 1 & 9\end{array}$
0,177

Escola6

$\begin{array}{lll}1 / 5 & 1 / 5 & 1 \\ 1 / 4 & 1 / 4 & 2\end{array}$

$1 / 2$

$\begin{array}{ll}1 / 8 & 1 / 4 \\ 1 / 7 & 1 / 3\end{array}$

$\begin{array}{ccc}1 & 9 & 0,157 \\ 1 / 4 & 6 & 0,047\end{array}$

0,157

$\begin{array}{cccc}3 & 4 & 8 & 7 \\ 1 / 2 & 1 & 4 & 3\end{array}$

Escola7

$\begin{array}{ccc}1 & 1 / 7 & 1 / 3 \\ 7 & 1 & 5\end{array}$

7

0,064

$\begin{array}{llll}1 & 5 & 9 & \mathbf{0 , 4 0 8}\end{array}$

\begin{tabular}{|c|c|c|c|c|c|}
\hline 1 & 4 & 3 & $1 / 5$ & 1 & 9 \\
\hline $1 / 9$ & $1 / 6$ & $1 / 7$ & $1 / 9$ & $1 / 9$ & 1 \\
\hline
\end{tabular}

\begin{tabular}{cccccccccc} 
Escola7 & $1 / 9$ & $1 / 9$ & $1 / 6$ & $1 / 7$ & $1 / 9$ & $1 / 9$ & 1 & 0,019 \\
\hline Conforto das Cadeiras & Escola 1 & Escola 2 & Escola 3 & Escola 4 & Escola 5 & Escola 6 & Escola 7 & PML's \\
\hline Escola1 & 1 & 1 & 1 & 2 & $1 / 2$ & 1 & $1 / 2$ & 0,118 \\
Escola2 & 1 & 1 & $1 / 2$ & 1 & $1 / 3$ & $1 / 2$ & $1 / 3$ & 0,078 \\
Escola3 & 1 & 2 & 1 & 3 & $1 / 2$ & 1 & 1 & 0,151 \\
Escola4 & $1 / 2$ & 1 & $1 / 3$ & 1 & $1 / 4$ & $1 / 3$ & $1 / 3$ & 0,060 \\
Escola5 & 2 & 3 & 2 & 4 & 1 & 2 & 1 & $\mathbf{0 , 2 4 6}$ \\
Escola6 & 1 & 2 & 1 & 3 & $1 / 2$ & 1 & 1 & 0,151 \\
Escola7 & 2 & 3 & 1 & 3 & 1 & 1 & 1 & 0,196 \\
\hline
\end{tabular}

Revista Produção Online, Florianópolis, SC, v.14, n. 4, p. 1433-1451, out./dez. 2014. 
Tabela 4 - Prioridades Médias Locais à luz das alternativas

(Conclusão)

\begin{tabular}{ccccccccc}
\hline Cantina & Escola 1 & Escola 2 & Escola 3 & Escola 4 & Escola 5 & Escola 6 & Escola 7 & PML's \\
\hline Escola1 & 1 & 5 & $1 / 2$ & $1 / 3$ & $1 / 7$ & $1 / 4$ & 6 & 0,074 \\
Escola2 & $1 / 5$ & 1 & $1 / 6$ & $1 / 8$ & $1 / 9$ & $1 / 9$ & 1 & 0,023 \\
Escola3 & 2 & 6 & 1 & $1 / 3$ & $1 / 7$ & $1 / 4$ & 6 & 0,090 \\
Escola4 & 3 & 8 & 3 & 1 & $1 / 5$ & $1 / 2$ & 8 & 0,152 \\
Escola5 & 7 & 9 & 7 & 5 & 1 & 4 & 9 & $\mathbf{0 , 4 3 1}$ \\
Escola6 & 4 & 9 & 4 & 2 & $1 / 4$ & 1 & 9 & 0,207 \\
Escola7 & $1 / 6$ & 1 & $1 / 6$ & $1 / 8$ & $1 / 9$ & $1 / 9$ & 1 & 0,023 \\
\hline Valor de Serviço & Escola 1 & Escola 2 & Escola 3 & Escola 4 & Escola 5 & Escola 6 & Escola 7 & PML's \\
\hline Escola1 & 1 & 1 & $1 / 4$ & $1 / 3$ & $1 / 3$ & 1 & 2 & 0,077 \\
Escola2 & 1 & 1 & $1 / 4$ & $1 / 3$ & $1 / 3$ & 1 & 2 & 0,077 \\
Escola3 & 4 & 4 & 1 & 2 & 2 & 4 & 6 & 0,329 \\
Escola4 & 3 & 3 & $1 / 2$ & 1 & 1 & 2 & 4 & 0,192 \\
Escola5 & 3 & 3 & $1 / 2$ & 1 & 1 & 2 & 4 & 0,192 \\
Escola6 & 1 & 1 & $1 / 4$ & $1 / 2$ & $1 / 2$ & 1 & 3 & 0,092 \\
Escola7 & $1 / 2$ & $1 / 2$ & $1 / 6$ & $1 / 4$ & $1 / 4$ & $1 / 3$ & 1 & 0,043 \\
\hline
\end{tabular}

\section{- Cálculo das Prioridades Globais (PG's)}

Após obter a PML de cada critério à luz do Foco Principal e as PMLs de cada escola à luz de cada critério, a prioridade global (PG) de uma escola $i$ pode ser obtida por meio da equação (3).

$$
P G_{a_{i}}=\left(P M L_{c_{1}} * P M L_{a_{i}}\right)+\left(P M L_{c_{\mathrm{n}}} * P M L_{a_{\mathrm{i}}}\right)+\cdots+\left(P M L_{c_{n}} * P M L_{a_{\mathrm{i}}}\right)
$$

As prioridades globais apresentadas na Tabela 5 revelam que $\mathbf{a}_{5}$ é a escola que apresenta melhor desempenho quando todos os critérios são considerados, seguida muito próxima pela escola $\mathbf{a}_{7}$. Apesar de $\mathbf{a}_{7}$ ter sido a melhor escola em sete subcritérios, em termos de conforto térmico (CT) e cantina (C) o nível de satisfação dos seus estudantes foi muito ruim. A escola $\mathbf{a}_{3}$ foi a melhor em termos de valor do serviço (VS) porque se trata de uma universidade que oferece cursos gratuitos.

Tabela 5 - Prioridades globais e síntese das prioridades médias locais

\begin{tabular}{|c|c|c|c|c|c|c|c|c|c|c|c|c|c|c|c|c|}
\hline & & \multicolumn{4}{|c|}{ Ensino } & \multicolumn{5}{|c|}{$\begin{array}{c}\text { Relacionamento } \\
\text { Interpessoal }\end{array}$} & \multicolumn{3}{|c|}{ Estrutura Física } & \multicolumn{3}{|c|}{$\begin{array}{l}\text { Valor do } \\
\text { Serviço }\end{array}$} \\
\hline & DP & $\mathbf{C P}$ & ELP & $\mathbf{P}$ & ME & QM & AS & EP & CA & LB & TM & CT & CC & C & VS & PG's \\
\hline$a_{1}$ & 0,16 & 0,20 & 0,15 & 0,09 & 0,15 & 0,20 & 0,15 & 0,15 & 0,14 & 0,15 & 0,23 & 0,18 & 0,12 & 0,07 & 0,08 & 0,14 \\
\hline $\mathbf{a}_{2}$ & 0,16 & 0,09 & 0,15 & 0,20 & 0,12 & 0,12 & 0,08 & 0,15 & 0,14 & 0,13 & 0,14 & 0,16 & 0,08 & 0,02 & 0,08 & 0,12 \\
\hline$a_{3}$ & 0,14 & 0,09 & 0,15 & 0,05 & 0,05 & 0,05 & 0,03 & 0,13 & 0,07 & 0,03 & 0,08 & 0,05 & 0,15 & 0,09 & 0,33 & 0,15 \\
\hline $\mathbf{a}_{4}$ & 0,16 & 0,09 & 0,07 & 0,05 & 0,15 & 0,07 & 0,32 & 0,15 & 0,04 & 0,08 & 0,08 & 0,06 & 0,06 & 0,15 & 0,19 & 0,14 \\
\hline$a_{5}$ & 0,18 & 0,17 & 0,18 & 0,22 & 0,20 & 0,20 & 0,18 & 0,15 & 0,33 & 0,22 & 0,16 & 0,41 & 0,25 & 0,43 & 0,19 & 0,20 \\
\hline$a_{6}$ & 0,05 & 0,08 & 0,06 & 0,05 & 0,05 & 0,04 & 0,08 & 0,09 & 0,12 & 0,13 & 0,05 & 0,13 & 0,15 & 0,21 & 0,09 & 0,08 \\
\hline$a_{7}$ & 0,16 & 0,28 & 0,25 & 0,33 & 0,27 & 0,32 & 0,17 & 0,17 & 0,17 & 0,25 & 0,27 & 0,02 & 0,20 & 0,02 & 0,04 & 0,18 \\
\hline
\end{tabular}

Revista Produção Online, Florianópolis, SC, v.14, n. 4, p. 1433-1451, out./dez. 2014. 
Neste contexto, os resultados apresentados podem ser explorados pelas escolas em duas vertentes principais: melhorar o desempenho à luz de subcritérios que ocasionam menor satisfação dos seus alunos, visando à manutenção destes; e, desenvolver ações para explorar as fraquezas dos concorrentes à luz de subcritérios que ocasionam menor satisfação daqueles alunos, visando à captação de alunos.

\section{- Verificação da Consistência dos julgamentos}

Segundo Saaty (2000), o método AHP permite determinar o grau de inconsistência dos julgamentos paritários avaliando a intensidade com que o autovalor da matriz de julgamentos se afasta de sua ordem. Para a execução dos cálculos, Saaty propôs um procedimento composto de algumas etapas, ilustradas por meio da matriz de critérios-Foco Principal:

a) Construção de uma matriz auxiliar $A$ " com os valores da matriz de julgamento multiplicado pelas PMLs de cada critério:

$$
A^{\mathrm{u}}=\left(\begin{array}{cccc}
1 *(0,438) & 2 *(0,246) & 5 *(0,070) & 2 *(0,246) \\
1 / 2 *(0,438) & 1 *(0,246) & 4 *(0,070) & 1 *(0,246) \\
1 / 5 *(0,438) & 1 / 4 *(0,246) & 1 *(0,070) & 1 / 4 *(0,246) \\
1 / 2 *(0,438) & 1 *(0,246) & 4 *(0,070) & 1 *(0,246)
\end{array}\right)=\left(\begin{array}{cccc}
0,438 & 0,492 & 0,350 & 0,492 \\
0,219 & 0,246 & 0,280 & 0,246 \\
0,088 & 0,062 & 0,070 & 0,062 \\
0,219 & 0,246 & 0,280 & 0,246
\end{array}\right)
$$

b) Soma dos elementos de cada linha de $A$ ", constituindo o vetor de prioridade $P^{\prime}$. Os valores resultantes serão divididos pelo valor da $\mathrm{PML}$ associada a esta linha, constituindo os elementos do vetor $P_{a u x}$. Os vetores obtidos são:

$$
P^{\mathrm{n}}=(1,772 ; 0,991 ; 0,282 ; 0,991) \text { e } P_{\operatorname{aux}}=\left(\frac{1,772}{0,438} ; \frac{0,991}{0,246} ; \frac{0,282}{0,070} \frac{0,991}{0,246}\right)=(4,046 ; 4,028 ; 4,009 ; 4,028)
$$

c) Cálculo do autovalor máximo por meio da média dos elementos de $P_{\text {aux }}: \lambda_{\max }=$ 4,03 .

d) Cálculo do Índice de Consistência $(I C)$ e da Razão de Consistência $(R C)$, sendo IR um índice de consistência para uma matriz recíproca, com elementos não negativos e gerada randomicamente. Para uma matriz de 
julgamentos de ordem 4, o valor de IR é 0,90. Segundo Saaty (2000) os julgamentos de uma matriz são consistentes quando $\mathrm{RC} \leq 0,10$.

$$
I C=\frac{\lambda_{M a ̊}-n}{(n-1)}=\frac{4,03-4}{(4-1)}=0,01 \text { e } R C=\frac{I C}{I R}=\frac{0,01}{0,90}=0,01
$$

Os valores de $R C$ para as demais matrizes de julgamento são apresentadas na Tabela 6 , sendo todas consideradas consistentes.

Tabela 6 - Índice de Consistência e Razão de Consistência à luz dos critérios e subcritérios

\begin{tabular}{lcc}
\hline Matriz & IC & RC \\
\hline Ensino (ED) & 0,01 & 0,01 \\
- Didática do professor (DP) & 0,01 & 0,01 \\
- Conhecimento do professor (CP) & 0,01 & 0,01 \\
- Evitar língua portuguesa (ELP) & 0,01 & 0,01 \\
- Pontualidade (P) & 0,01 & 0,01 \\
- Método de ensino (ME) & 0,01 & 0,01 \\
- Qualidade do material (QM) & 0,01 & 0,01 \\
Relacionamento Interpessoal (RI) & 0,00 & 0,01 \\
- Atendimento na secretaria (AS) & 0,02 & 0,02 \\
- Educação do professor (EP) & 0,01 & 0,01 \\
- Convivência agradável (CA) & 0,01 & 0,01 \\
Estrutura Física (EF) & 0,02 & 0,03 \\
- Laboratório (LB) $\quad 0,01$ & 0,01 \\
- Cantina (C) & 0,11 & 0,08 \\
- Sala de Aula (SA) & 0,00 & 0,00 \\
- Tamanho da sala (TM) & 0,01 & 0,01 \\
- Conforto térmico (CT) & 0,10 & 0,07 \\
- Conforto das cadeiras (CC) & 0,02 & 0,01 \\
Valor do Serviço (VS) & 0,01 & 0,01 \\
\hline
\end{tabular}

\subsection{Emprego da Média Ponderada e comparação dos resultados}

Neste estudo, a mensuração da qualidade das escolas de idiomas $\left(Q_{s}\right)$ por meio da média ponderada foi realizada a partir do emprego da equação (4): 


$$
Q_{S}\left(a_{i}\right)=\sum_{k=1}^{r} \sum_{j=1}^{n} G l_{j k} G S_{j k}\left(a_{i}\right) / \sum_{k=1}^{r} \sum_{j=1}^{n} G l_{j k}
$$

Onde:

- $r$ é o $\mathrm{n}^{0}$ de avaliadores de cada escola;

- $n$ é o no de subcritérios;

- $G I_{j k}$ é o Grau de Importância do subcritério $j$ segundo a percepção do avaliador $k$;

- $G S\left(a_{i}\right)_{j k}$ é o Grau de Satisfação com a escola $a_{i}$ à luz do critério $j$, segundo a percepção do avaliador $k$.

A Tabela 7 mostra o resultado da comparação dos métodos AHP e Média Ponderada, onde se observa inversões nas ordenações das escolas $\mathbf{a}_{1}, \mathbf{a}_{2}, \mathbf{a}_{3}$ e $\mathbf{a}_{7}$. Porém, as escolas $\mathbf{a}_{4}, \mathbf{a}_{5}$ e $\mathbf{a}_{6}$ tiveram a mesma ordem nos dois métodos. Em ambos os métodos a escola $\mathbf{a}_{5}$ obteve melhor ordem.

Tabela 7 - Comparação da ordenação nos métodos

\begin{tabular}{ccc}
\hline Escolas & Ordenação pela Média Ponderada (Qs) & Ordenação no Método AHP (PGs) \\
\hline Escola 1 & $2^{\circ}(8,43)$ & $4^{\circ}(0,14)$ \\
Escola 2 & $4^{\circ}(8,16)$ & $6^{\circ}(0,12)$ \\
Escola 3 & $6^{\circ}(7,70)$ & $3^{\circ}(0,15)$ \\
Escola 4 & $5^{\circ}(7,78)$ & $5^{\circ}(0,14)$ \\
Escola 5 & $\mathbf{1}^{\circ}(8,89)$ & $\mathbf{1}^{\circ}(0,20)$ \\
Escola 6 & $7^{\circ}(7,61)$ & $7^{\circ}(0,08)$ \\
Escola 7 & $3^{\circ}(8,41)$ & $2^{\circ}(0,18)$ \\
\hline
\end{tabular}

Apesar de permitir a análise da consistência dos julgamentos - uma virtude em relação a outros métodos, o emprego do AHP pode se tornar exaustivo em problemas com muitas alternativas e critérios. No estudo em questão, mesmo com os valores da importância dos critérios e do grau de satisfação, o decisor precisou realizar 345 julgamentos paritários para selecionar a escola de idiomas.

A Média Ponderada se mostrou mais simples de ser aplicado, pois apenas os valores médios da importância dos critérios e da satisfação com as escolas à luz dos critérios foram considerados (valores fornecidos pelos estudantes das escolas de idiomas). Nenhum julgamento foi realizado pelo decisor, embora ele próprio pudesse atribuir a importância dos critérios. Por outro lado, a média aritmética é um método compensatório, ou seja, uma alternativa pode ter um desempenho muito ruim em

Revista Produção Online, Florianópolis, SC, v.14, n. 4, p. 1433-1451, out./dez. 2014. 
determinado critério e ter esse desempenho "compensado" por um desempenho muito bom em outro critério. Após somar os valores, uma eventual fraqueza da alternativa pode não ser percebida. No AHP, as prioridades médias indicam as alternativas que se destacam à luz de cada critério.

Considera-se que a escolha por um dos dois métodos dependerá da relevância do problema decisório e também da disponibilidade e interesse do decisor em realizar os julgamentos paritários do AHP, embora esse método seja mais bem fundamentado cientificamente e permita análises diferenciadas.

\section{CONSIDERAÇÕES FINAIS}

A escolha de uma escola de idiomas tem sido um dos problemas decisórios mais comuns na atualidade, diante da necessidade cada vez mais evidente de ter conhecimento de, ao menos, um idioma estrangeiro. Observa-se que a escolha de escolas de idiomas tem sido em função de informações boca-a-boca (amigos, pessoas conhecidas, dentre outras), marketing das escolas e experimentações sem critério - fato que será investigado em estudo posterior.

Desejando contribuir para este problema, este artigo apresentou um estudo no qual foram empregados dois métodos de apoio à tomada de decisão (AHP e Média Ponderada) com o objetivo de escolher uma escola de idiomas. Para tanto, foram considerados os dados de um estudo anterior, no qual foram captados o grau de importância dos critérios e o grau de satisfação de estudantes de graduação e pós-graduação em relação às escolas de idiomas que frequentavam.

A escola de idiomas mais bem ordenada foi a mesma em ambos os métodos de AMD, embora algumas escolas tenham obtido posições diferentes. A estrutura hierárquica, as prioridades locais e a verificação da consistência dos julgamentos são virtudes do método AHP frente à Média Ponderada. Porém, a grande quantidade de julgamentos paritários necessária ao estudo demandou atenção e significativo esforço cognitivo do decisor, além de maior tempo para realização das análises. Estes aspectos podem favorecer a preferência pela Média Ponderada em estudos similares.

Vale ressaltar que a modelagem decisória empregada neste artigo pode ser utilizada não somente por pessoas interessadas em escolher uma escola de idiomas, mas também por empresas que desejam escolher uma escola de idiomas

Revista Produção Online, Florianópolis, SC, v.14, n. 4, p. 1433-1451, out./dez. 2014. 
para seus funcionários no contexto de um programa de capacitação/gestão de pessoas. Mais ainda, escolas de idiomas podem utilizar a abordagem para mensurar a qualidade da escola percebida por seus estudantes (autoavaliação) e também para mensurar a qualidade das escolas concorrentes, conforme percebida pelos estudantes das mesmas. Estas vertentes visam atingir dois objetivos principais: melhorar o desempenho da escola à luz de subcritérios que ocasionam menor satisfação dos seus alunos (ações para manutenção de alunos) e desenvolver ações para explorar as fraquezas dos concorrentes à luz de subcritérios que ocasionam menor satisfação daqueles alunos (ações para captação de alunos).

\section{REFERÊNCIAS}

BENTES, A. V.; CARNEIRO, J.; DA SILVA, J. F.; KIMURA, H. Multidimensional assessment of organizational performance: Integrating BSC and AHP. Journal of Business Research, V.65, n.12, p.1790-1799, 2012.

http://dx.doi.org/10.1016/j.jbusres.2011.10.039

COSTA, H. G. IPÊ 1.0: Guia do Usuário. Niterói: UFF, 2004. Disponível em $<$ http://www.producao.uff.br/conteudo/rpep/volume42004/RelPesq V4 2004 05.pdf> . Acesso em 23 fev. 2013.

FREITAS, A. L. P.; BATISTA, V. R.; ALMEIDA, G. M. M. Qualidade de serviços em escolas de idiomas: um estudo exploratório. In: ENEGEP, 32., 2012, Bento Gonçalves. Anais... Rio de Janeiro: ABEPRO, 2012. p. 1 - 13.

FREITAS, A. L. P.; MORAIS, A. S. C. Avaliar estabelecimentos de hospedagem face à informação disponível nas homepages. Revista Portuguesa e Brasileira de Gestão (Lisboa), v. 9, p.20-37, 2010.

FREITAS, A. L. P.; TREVIZANO, W. A.; COSTA, H. G. Uma abordagem multicritério para problemas decisórios com múltiplos grupos de avaliadores. Revista Investigação Operacional, Lisboa, n. 28, p.133-149, 2008.

GOMES, C. F. S.; COSTA, H. G. Abordagem estratégica para a seleção de sistemas ERP utilizando apoio multicritério à decisão. Revista Produção Online, Florianópolis, SC, v.13, n. 3, p. 1060-1088, jul./set. 2013. http://dx.doi.org/10.14488/1676-1901.v13i3.1385

KRISHNAMURTHY, D.; ROLIA, J.; XU, M. WAM-The Weighted Average Method for Predicting the Performance of Systems with Bursts of Customer Sessions. IEEE Transactions on Software Engineering, v. 37 n. 5, p. 718 -735, 2011. http://dx.doi.org/10.1109/TSE.2011.65

LAÑEZ, M. M.; CUNHA, C. B. Uma metodologia para a seleção de um provedor de serviços logísticos. Produção, São Paulo, v. 16, n. 3, p.394-412, 2006.

Revista Produção Online, Florianópolis, SC, v.14, n. 4, p. 1433-1451, out./dez. 2014. 
RAFAELI, L.; MULLER, C. J. Estruturação de um índice consolidado de desempenho utilizando o AHP. Gestão e Produção, São Carlos, v. 14, n. 2, p.363377, 2007.

RATHNAM, G. Interaction effects of consumers' product class knowledge and agent search strategy on consumer decision making in electronic commerce. IEEE Transactions on systems, man, and cybernetics - Part A: Systems and Humans, v. 35, n. 4, July 2005. http://dx.doi.org/10.1109/TSMCA.2005.850606

SAATY, T. L. Método de análise hierárquica. Tradução e revisão técnica Wainer da Silveira e Silva. São Paulo: Makron Books, 1991.

SAATY, T. L. Decision making for leaders. Pittsburg, USA: WS. Publications, 2000.

SOUZA, D. V. S.; KLIEMANN NETO, F. J.; ANZANELLO, M. J. Avaliação de desempenho da cadeia de suprimentos balizada por critérios de competitividade empresarial. Revista Produção Online, Florianópolis, SC, v.12, n. 3, p. 756-778, jul./set. 2012. http://dx.doi.org/10.14488/1676-1901.v12i3.976

TINOCO, M. A. C. Proposta de modelos ampliados de satisfação de clientes. 2011. 173 f. Tese (Doutorado) - Curso de Engenharia de Produção, Universidade Federal do Rio Grande do Sul, Porto Alegre, 2011. Cap. 6.

TREVIZANO, W. A.; FREITAS, A. L. P. Emprego do Método da Análise Hierárquica (AHP) na seleção de processadores. In: ENEGEP, 25., 2005, Porto Alegre. Anais.... Rio de Janeiro: Abepro, 2005. p. 2972 - 2979.

VINCKE, P. L'aide multicritère à la decision. Editions de l'Université de Bruxelles Ed. Ellipses, 1989.

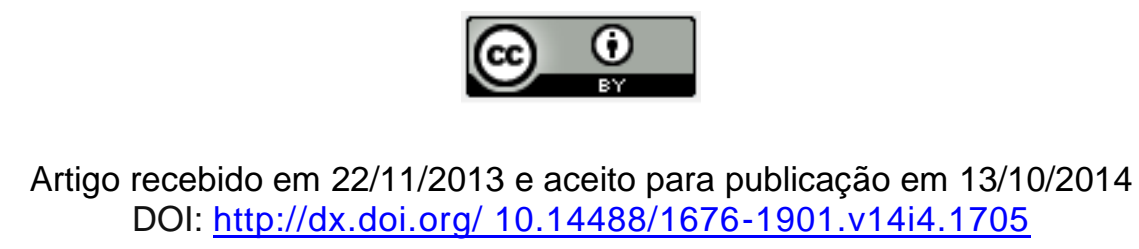

DOI: http://dx.doi.org/ 10.14488/1676-1901.v14i4.1705 